\title{
Um Estudo Sobre os Impactos da Surpresa da Política Monetária na
} Atividade Econômica Brasileira ${ }^{1}$

\author{
Ricardo da Cruz Gouveia Vieira ${ }^{2}$ \\ Carlos Eduardo Soares Gonçalves ${ }^{3}$
}

\begin{abstract}
RESUMO
Este trabalho estuda os impactos da política monetária nas variáveis reais da economia brasileira. Será testado o efeito de movimentos não esperados da política monetária sobre a atividade econômica e o desemprego com base nos modelos Neoclássicos e NovoKeynesianos, de que o que importa para o comportamento das variáveis reais é a parte nãoesperada da política monetária. Como medida de surpresa da política monetária será utiliza o erro de previsão do mercado para a taxa de juros estabelecida pela autoridade monetária.

Palavras Chaves: Política Monetária, Brasil, Taxa de Juros

Classificação JEL : E43 e E52
\end{abstract}

\begin{abstract}
This work studies the impacts of the monetary policy in the real variables of the Brazilian economy. It will be tested the unanticipated movements of the monetary policy in the economic activity and unemployment according whit the Neoclassical and new Keynesians models, which the importance the real variables is the unanticipated component of the monetary policy. As measure of surprise it will be used the error forecast for the interest rate established by the monetary authority.
\end{abstract}

Key Words: Monetary Policy, Brazil, Interest Rate

JEL Classification: E43 e E52

\footnotetext{
1 Artigo baseado na dissertação de mestrado do primeiro autor sob a orientação do co-autor e com suporte financeiro da CAPES.

${ }^{2}$ Mestrando em Teoria Econômica pela IPE/FEA/USP. Rua Oliviera Dias, 380 - São Paulo, SP. CEP: 01433 030. E-mail: ricardocgv@yahoo.com.br.

3 Professor do Departamento de Economia da FEA/USP. Av. Prof. Luciano Gualberto, 908 - Cidade Universitária, São Paulo, SP. CEP: 05508-900. E-mail: cesg@usp.br
} 


\section{INTRODUÇÃO}

Pelo menos desde David Hume, os economistas vêm debatendo a relação entre moeda e as variáveis reais da economia. Em particular, muito da pesquisa empírica desde o final dos anos 50 tem buscado investigar a relação entre moeda e atividade econômica. O "Consenso Keynesiano", que dominou o debate macroeconômico durante as décadas de 50 e 60, defendia a não neutralidade da moeda. Essa abordagem sofreu severas críticas no inicio dos anos 70 , tanto no plano teórico, com a revolução das expectativas racionais ${ }^{4}$, como no plano empírico, dado que o choque do petróleo havia levado a um desaparecimento da tão alardeada curva de Phillips.

Assim, a idéia de um tradeoff permanente entre inflação e desemprego (ou atividade) foi substituída pela nova curva de Phillips aumentada pelas expectativas. Segundo esta formulação, apenas surpresas nominais (inflação acima da inflação esperada) teriam algum impacto sobre a atividade. Aqui vale ressaltar que os próprios modelos originados na "contrarevolução" novo-keynesiana dos anos 80 chegam a este mesmo resultado: surpresas monetárias afetariam o nível de produção, mas a parte esperada da moeda influenciaria apenas o nível de preços (ver Romer (2001)).

Em dois artigos seminais, o economista Robert Barro ${ }^{5}$ leva para a arena empírica a idéia de que a parte não-esperada da moeda influencia a atividade. Usando os resíduos de uma regressão onde a variável explicada é a moeda (os resíduos refletindo a parte não-esperada da moeda), o autor encontra evidências favoráveis à tese de que a parte não esperada da moeda

\footnotetext{
${ }^{4}$ Lucas (1973)

${ }^{5}$ Barro (1977, 1978).
} 
afeta a atividade. Interessantemente, Frederic Mishkin (1982) repete o experimento de Barro e encontra evidencias de que, além da parte não-esperada, a parte esperada da moeda também apresenta correlação positiva com a atividade econômica. Neste artigo, de natureza eminentemente empírica, nos baseamos nos testes realizados por estes dois autores para investigar a relação entre política monetária (parte esperada e não-esperada) e atividade no Brasil pós-flutuação cambial. A única diferença crucial deste artigo para os acima mencionados é que nossa medida de política monetária é dada pela taxa de juros, e não por medidas de agregados monetários.

O trabalho está dividido em quatro seções, incluindo esta introdução. A segunda discutirá a estratégia empírica e a base de dados, a terceira apresentará os resultados obtidos, e a quarta finalmente conclui o trabalho. 


\section{DADOS E METODOLOGIA}

Nesta seção serão expostos os dados e a metodologia que utilizamos para o desenvolvimento do trabalho.

Nossa base de dados incorpora o período 1999\%2005. A idéia de empregarmos dados referentes apenas ao período de flutuação cambial é minimizar a importância das conhecidas críticas a respeito da presença de quebras estruturais nos dados. Por trabalharmos com um período de tempo relativamente reduzido, optamos por usar dados de freqüência mensal. As séries empregadas foram: (i) taxa de desemprego (Seade e Dieese/PED), (ii) horas trabalhadas da CNI, (iii) produção industrial (IBGE), (iv) inflação mensal com base no $\operatorname{IPCA},(v)$ expectativa de inflação (pesquisa Focus) ${ }^{7},(v i)$ taxa de juros de curto prazo (Selic) e, (vii) taxa de juros de longo prazo (swaps pré-DI de 30 e 360 dias).

Antes de tudo, para testarmos a influência da surpresa monetária na atividade, precisamos construir inicialmente uma medida de surpresa. Nossa primeira medida de quanto os agentes privados são surpreendidos por decisões de política monetária é simples: calculamos a diferença entre as taxas de juros de um dado instrumento financeiro (no caso os swaps de 30 e 360 dias) negociadas um dia depois, e um dia antes, da decisão mensal do Comitê de Política Monetária (Copom). A diferença entre as taxas negociadas para um mesmo instrumento antes e depois do Copom é uma medida clara de quanto o mercado foi (ou não) surpreendido pela decisão do BC.

\footnotetext{
${ }^{6} \mathrm{Na}$ verdade a base começa apenas em setembro de 1999, quando se iniciam as reuniões sistemáticas do Comitê de Política Monetária.

${ }^{7}$ Série que se inicia apenas em janeiro de 2000.
} 
Em notação, esta primeira medida de resíduo é portanto dada por : $R J=r_{t}^{Q}-r_{t}^{T}$,

Onde:

$R J$ é o resíduo de juros; $r_{t}^{Q}$ é a taxa de juros na quinta-feira (maturidade $t$ ), um dia após o Banco Central do Brasil definir a taxa de juros básica da economia; e $r_{t}^{T}$ é a taxa de juros esperada pelo mercado um dia antes do Banco Central determinar a taxa básica de juros.

Construímos duas medidas de surpresa com base nesta metodologia, uma empregando taxas de juros de mercado de maturidade de 30 dias e outra mais longa, com maturidade de 360 dias.

A segunda medida de surpresa monetária se assemelha mais à idéia dos já mencionados artigos de Robert Barro (com a diferença de que este autor emprega a própria moeda como medida de instrumento, e nós empregamos a taxa de juro): ela é o resíduo de uma Regra de Taylor onde a variável explicada é o juro básico, e as explicativas são juros defasados, atividade e expectativa de inflação. A seguir, apresentamos os resultados de nossa estimação da Regra de Taylor ${ }^{8}$. 


\begin{tabular}{lc}
\hline \multicolumn{2}{c}{ Variável Dependente: Taxa de Juros (Jan/2000 - Out/2005) } \\
\hline & $2,585 \quad * *$ \\
Constante & 1,211 \\
Desvio-padrão & $0,472 \quad * * *$ \\
(Expectativa de Inflação - Meta) & 0,125 \\
Desvio-padrão & 1,126 \\
Hiato do Produto (Filtro HP) & 7,510 \\
Desvio-padrão & $0,839 \quad * * *$ \\
Juros (-1) & 0,066 \\
Desvio-padrão & 70 \\
& 0,888 \\
\hline Observações & 0,215 \\
$\mathrm{R}^{2}$ & \\
Teste LM B-G & \\
\hline \hline Sendo que *** e *** significa a variável é estatisticamente significante a 10\%, 5\% e \\
1\%, respectivamente. Teste LM B-G (Breusch -Godfrey) de correlação serial, para a \\
hipótese nula de não correlação serial (P-valor).
\end{tabular}

Mais uma vez, os resíduos da regressão acima constituem nossa segunda medida de surpresa de política monetária. É interessante observarmos no gráfico de dispersão abaixo que as duas diferentes medidas de surpresa são altamente correlacionadas quando excluímos três claros outliers (crise do "apagão" de 2001, e duas observações no período das tensões eleitorais de 2002) do total de 70 dados de nossa amostra (correlação simples de 0,70 - Figura 1).

Além do gráfico de dispersão, podemos observar simultaneamente como as três séries dos resíduos obtidas se comportam (Figura 2). Neste gráfico, ficam em evidência os autliers de 2001 e de 2002.

\footnotetext{
${ }^{8}$ Ver Taylor $(1993,1999)$.
} 
Figuras 1. Dispersão entre Resíduo de 30 Dias e Resíduo da Regra de Taylor Com todas as observações $(E)$ e sem três observações outlier $(D)$
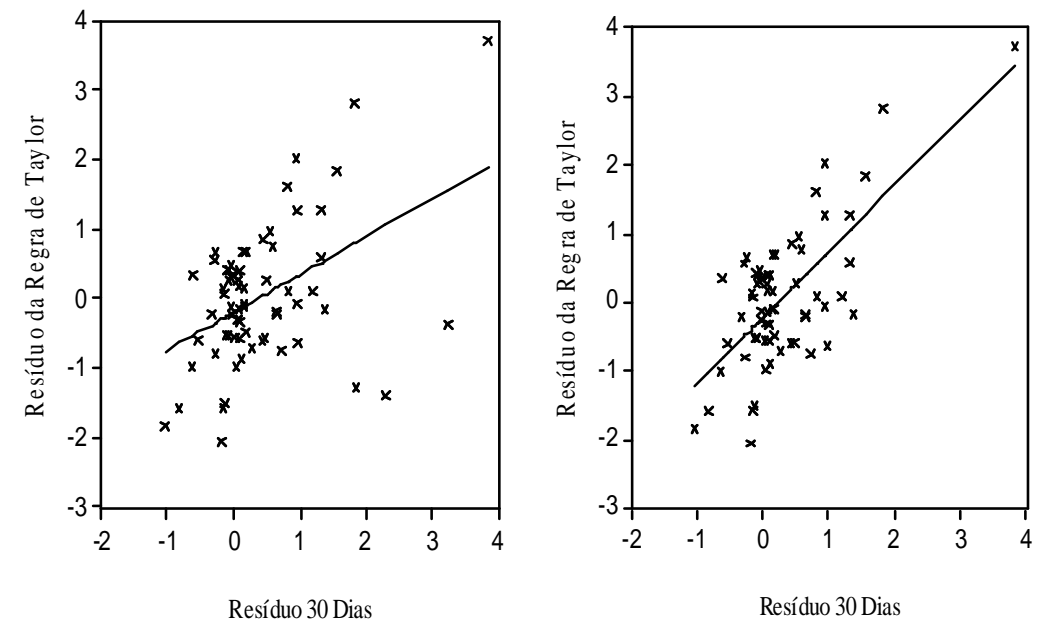

Figura 2. Séries dos Resíduos da Política Monetária (Set/1999 a Out/2005).

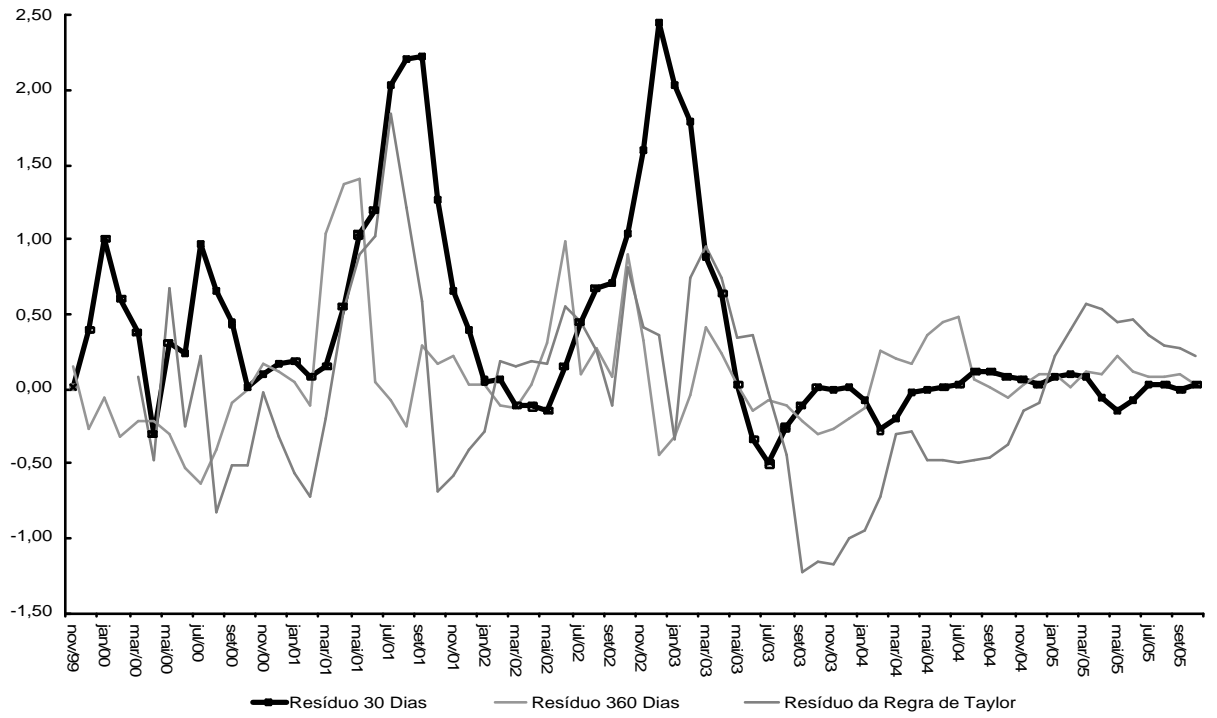

Nossa estratégia básica será então regredir medidas de atividade econômica como desemprego, variação nas horas trabalhadas e variação na produção em suas defasagens, nas medidas de surpresa monetária, e também na parte esperada da política monetária. 
Em notação:

$$
y_{t}=\alpha_{0}+\sum_{i=1}^{N} \omega_{i} y_{t-i}+\sum_{i=0}^{N} \beta_{i} x_{t-i}+\sum_{i=0}^{N} \delta_{i} x_{t-i}^{e}+e_{t}
$$

Onde:

$y_{t}$ é desemprego, horas trabalhadas ou produção no período; $x_{t-i}$ é a surpresa da política monetária; $x_{t-i}^{e}$ é a parte esperada da política monetária, $e_{t}$ é um termo estocástico, com suas propriedades usuais, e $N$ é no máximo igual a 12.

As regressões serão estimadas com até doze defasagens da variável dependente, com a intenção de corrigir a correlação serial dos resíduos ${ }^{9}$. Depois de detectada a não existência e correlação serial através do teste $L M$ Breusch/Godfrey, será selecionado a melhor regressão através dos critérios de informação tradicionais ${ }^{10}$.

Em um segundo passo, serão feitas estimações de regressões degeneradas, ou seja, serão estimados modelos com apenas uma defasagem de cada parte, esperada e não esperada dos juros $^{11}$. Por fim, todas nossas regressões foram rodadas empregando médias-móveis trimestrais com intuito de suavizar eventuais oscilações de curto prazo nas séries.

Dado que trabalharemos com três séries distintas de resíduo, temos três tipos de modelos para cada variável dependente. São eles:

\footnotetext{
${ }^{9}$ Ver Greene (2003), Davidson e MacKinnon (2004) e Favero (2001).

${ }^{10}$ Para a escolha do melhor modelo de defasagem foram analisados dois critérios de seleção, o Critério de Informação de Akaike (CIA) e o Critério de Informação de Schwarz (CIS).

${ }^{11}$ Os mesmos critérios de informação para a escolha do melhor modelo estimado serão utilizados nessa etapa.
} 
Modelo 1: $R J^{30}=r_{t}^{Q}-r_{t-2}^{T}$, diferença da equação para juros de 30 dias.

Modelo 2: $R J^{360}=r_{t}^{Q}-r_{t-2}^{T}$, diferença da equação para juros de 360 dias.

Modelo 3: $i_{t}=g_{0}+\beta i_{t-1}+g_{\pi} \pi_{t}+g_{t} x_{t}+\varepsilon_{t}$, resíduo da Regra de Taylor. 


\section{RESULTADOS}

Nesta seção, discutimos os resultados encontrados nas regressões feitas a partir da metodologia s ugerida na seção anterior. Os três primeiros modelos estimados estão reportados na tabela 1, onde a produção é a variável dependente e todas as defasagens são empregadas como regressores. Ou seja, surpresa de política monetária e também sua parte esperada entram com até doze meses de defasagem.

Como se pode ver, no modelo 1 várias defasagens são significativas e possuem o coeficiente com o sinal esperado. Além disso, é interessante notar a concentração dos coeficientes significantes nas seis primeiras defasagens. Diferentemente do modelo 1, no qual encontramos 6 dos $13 \beta^{\prime} s$ (parte não esperada) significantes, e também 6 dos $13 \delta$ 's(parte esperada) significantes, nos modelos 2 e 3 a maioria dos coeficientes não são estatisticamente diferentes de zero, mas os significativos mais uma vez se concentram nas defasagens mais curtas.

Já na tabela 2, onde não são incluídas todas as defasagens, obtivemos nos três modelos estimados coeficientes significativos e com o sinal esperado, tanto para a parte não esperada, quanto para a parte esperada dos juros.

Na tabela 3, onde a variável dependente é horas trabalhadas, não encontramos boas evidências dos efeitos da política monetária. No modelo 1 nenhum coeficiente se mostrou estatisticamente significativo, enquanto no modelo 2 apenas três coeficientes são estatisticamente significativos, sendo dois da parte não-esperada (com o sinal "errado" e significativos apenas a 10\%). Na tabela 4, com estimações com apenas algumas defasagens incluídas, os resultados são, contudo, um pouco melhores. 
Tabela 2. Resultado - Produção Industrial

\begin{tabular}{|c|c|c|c|c|c|c|c|c|c|}
\hline \multicolumn{10}{|c|}{ Variável Dependente: Produção Industrial com Média Móvel (Set/1999-Out/2005) } \\
\hline & \multicolumn{3}{|c|}{ Modelo 1} & \multicolumn{3}{|c|}{ Modelo 2} & \multicolumn{3}{|c|}{ Modelo 3} \\
\hline $\mathrm{a}_{0}$ & $-0,802$ & $(0,846)$ & & 0,332 & $(0,806)$ & & $-0,340$ & $(1,094)$ & \\
\hline$?_{1}$ & 0,638 & $(0,171)$ & $* * *$ & 0,752 & $(0,193)$ & $* * *$ & 0,539 & $(0,164)$ & $* * *$ \\
\hline$?_{2}$ & 0,050 & $(0,200)$ & & 0,030 & $(0,225)$ & & 0,137 & $(0,196)$ & \\
\hline$?_{3}$ & $-1,440$ & $(0,252)$ & $* * *$ & $-0,881$ & $(0,238)$ & $* * *$ & $-0,540$ & $(0,146)$ & $* * *$ \\
\hline$?_{4}$ & 0,729 & $(0,279)$ & $* *$ & 0,186 & $(0,301)$ & & & & \\
\hline$? 5$ & 0,213 & $(0,190)$ & & 0,085 & $(0,309)$ & & & & \\
\hline$? 6$ & $-0,925$ & $(0,252)$ & $* * *$ & $-0,369$ & $(0,302)$ & & & & \\
\hline$?_{7}$ & 0,428 & $(0,193)$ & $* *$ & $-0,019$ & $(0,307)$ & & & & \\
\hline$?_{8}$ & & & & 0,071 & $(0,278)$ & & & & \\
\hline$? 9$ & & & & $-0,362$ & $(0,259)$ & & & & \\
\hline$?$ & & & & 0,236 & $(0,209)$ & & & & \\
\hline$B_{0}$ & $-1,873$ & $(0,751)$ & $* *$ & 0,298 & $(0,471)$ & & 0,261 & $(0,313)$ & \\
\hline$B_{1}$ & 3,899 & $(1,411)$ & $* * *$ & $-0,144$ & $(0,563)$ & & $-1,103$ & $(0,804)$ & \\
\hline$B_{2}$ & $-2,949$ & $(1,377)$ & $* *$ & 0,274 & $(0,581)$ & & 0,933 & $(1,503)$ & \\
\hline$B_{3}$ & 0,039 & $(1,203)$ & & $-1,107$ & $(0,634)$ & $*$ & 0,426 & $(1,641)$ & \\
\hline$B_{4}$ & 0,113 & $(1,287)$ & & 0,509 & $(0,676)$ & & $-2,774$ & $(1,745)$ & \\
\hline$B_{5}$ & 1,810 & $(1,305)$ & & $-0,225$ & $(0,619)$ & & 2,304 & $(2,042)$ & \\
\hline$B_{6}$ & $-2,873$ & $(1,298)$ & $* *$ & $-0,274$ & $(0,625)$ & & 0,729 & $(1,976)$ & \\
\hline$B_{7}$ & 2,133 & $(1,336)$ & & $-0,293$ & $(0,593)$ & & $-2,374$ & $(1,967)$ & \\
\hline$B_{8}$ & $-0,893$ & $(1,233)$ & & 0,592 & $(0,581)$ & & 1,307 & $(1,978)$ & \\
\hline$B_{9}$ & 0,187 & $(1,224)$ & & $-0,441$ & $(0,563)$ & & 1,214 & $(1,644)$ & \\
\hline$\beta_{10}$ & $-1,104$ & $(1,172)$ & & 0,346 & $(0,463)$ & & $-2,801$ & $(1,574)$ & $*$ \\
\hline$B_{11}$ & 2,340 & $(1,128)$ & $* *$ & 0,587 & $(0,411)$ & & 0,881 & $(1,509)$ & \\
\hline$\beta_{12}$ & $-1,149$ & $(0,509)$ & $* *$ & $-0,284$ & $(0,371)$ & & 0,485 & $(0,779)$ & \\
\hline $\mathrm{d}_{0}$ & 1,086 & $(0,422)$ & $* *$ & 0,249 & $(0,222)$ & & 1,153 & $(0,848)$ & \\
\hline $\mathrm{d}_{1}$ & $-2,022$ & $(0,970)$ & $* *$ & $-0,459$ & $(0,492)$ & & $-2,554$ & $(2,428)$ & \\
\hline $\mathrm{d}_{2}$ & 0,312 & $(1,051)$ & & 0,229 & $(0,546)$ & & 0,359 & $(3,264)$ & \\
\hline $\mathrm{d}_{3}$ & 0,787 & $(1,069)$ & & 0,146 & $(0,503)$ & & 4,489 & $(3,181)$ & \\
\hline $\mathrm{d}_{4}$ & 1,563 & $(1,330)$ & & $-0,325$ & $(0,538)$ & & $-6,177$ & $(3,713)$ & $*$ \\
\hline$d_{5}$ & $-4,168$ & $(1,461)$ & $* * *$ & 0,220 & $(0,571)$ & & 2,067 & $(4,009)$ & \\
\hline $\mathrm{d}_{6}$ & 3,240 & $(1,332)$ & $* *$ & $-0,469$ & $(0,558)$ & & 3,348 & $(3,774)$ & \\
\hline $\mathrm{d}_{7}$ & $-0,328$ & $(1,157)$ & & 0,881 & $(0,574)$ & & $-4,525$ & $(3,924)$ & \\
\hline $\mathrm{d}_{8}$ & $-1,096$ & $(1,090)$ & & $-0,900$ & $(0,572)$ & & 0,878 & $(3,552)$ & \\
\hline $\mathrm{d}_{9}$ & 0,316 & $(1,134)$ & & 0,460 & $(0,503)$ & & 3,843 & $(3,002)$ & \\
\hline $\mathrm{d}_{10}$ & 1,980 & $(1,244)$ & & $-0,026$ & $(0,431)$ & & $-3,819$ & $(3,096)$ & \\
\hline $\mathrm{d}_{11}$ & $-2,900$ & $(1,104)$ & $* * *$ & $-0,283$ & $(0,370)$ & & 0,614 & $(2,364)$ & \\
\hline $\mathrm{d}_{12}$ & 1,294 & $(0,432)$ & $* * *$ & 0,278 & $(0,177)$ & & 0,352 & $(0,823)$ & \\
\hline Observações & 60 & & & 60 & & & 56 & & \\
\hline $\mathrm{R}^{2}$ & 0,877 & & & 0,866 & & & 0,869 & & \\
\hline Teste LM B-G & 0,828 & & & 0,402 & & & 0,569 & & \\
\hline
\end{tabular}

Sendo que *,** e*** significa a variável é estatisticamente significante a $10 \%, 5 \%$ e $1 \%$, respectivamente,

Teste LM B-G (Breusch-Godfrey) de correlação serial, para a hipótese nula de não correlação serial (P-valor). 
Tabela 3. Resultado - Produção Industrial (Degenerado)

\begin{tabular}{|c|c|c|c|c|c|c|c|c|c|}
\hline \multicolumn{10}{|c|}{ Variável Dependente: Produção Industrial com Média Móvel (Set/1999-Out/2005) } \\
\hline & \multicolumn{3}{|c|}{ Modelo 1} & \multicolumn{3}{|c|}{ Modelo 2} & \multicolumn{3}{|c|}{ Modelo 3} \\
\hline$a_{0}$ & 1,395 & $(0,544)$ & $* *$ & 1,098 & $(0,487)$ & $* *$ & 1,463 & $(0,574)$ & $* *$ \\
\hline$?_{1}$ & 0,480 & $(0,102)$ & $* * *$ & 0,728 & $(0,118)$ & $* * *$ & 0,522 & $(0,106)$ & $* * *$ \\
\hline$?_{2}$ & 0,093 & $(0,118)$ & & 0,034 & $(0,121)$ & & 0,061 & $(0,128)$ & \\
\hline$?_{3}$ & $-0,537$ & $(0,101)$ & $* * *$ & $-0,738$ & $(0,120)$ & $* * *$ & $-0,601$ & $(0,112)$ & $* * *$ \\
\hline$?_{4}$ & & & & 0,312 & $(0,121)$ & $* *$ & & & \\
\hline$B_{1}$ & $-0,382$ & $(0,112)$ & $* * *$ & & & & $-0,430$ & $(0,134)$ & $* *$ \\
\hline$\beta_{2}$ & & & & $-0,382$ & $(0,192)$ & $* *$ & & & \\
\hline $\mathrm{d}_{1}$ & $-0,050$ & $(0,028)$ & $*$ & & & & $-0,061$ & $(0,029)$ & $* *$ \\
\hline $\mathrm{d}_{2}$ & & & & $-0,042$ & $(0,022)$ & $* *$ & & & \\
\hline Observações & 71 & & & 70 & & & 67 & & \\
\hline $\mathrm{R}^{2}$ & 0,627 & & & 0,636 & & & 0,608 & & \\
\hline Teste LM B-G & 0,051 & & & 0,372 & & & 0,294 & & \\
\hline
\end{tabular}

Quando a variável dependente é a taxa de desemprego, encontramos resultados satisfatórios para os três modelos (tabela 5). No modelo 1 a resposta ocorreu nas últimas defasagens (na última para a parte não-esperada e na última e penúltima na parte esperada). Nos outros dois modelos os resultados se mostraram muito parecidos, acumulando os coeficientes significativos nas primeiras defasagens.

Nos modelos na forma degenerada (tabela 6) encontramos coeficientes significantes e com o sinal esperado, porém com defasagens distintas. Nos modelos 1 e 3 tem-se uma resposta mais rápida - na quarta e na terceira - enquanto no modelo 2, a décima defasagem é a significativa. 
Tabela 4. Resultado-Horas Trabalhadas

\begin{tabular}{|c|c|c|c|c|c|c|c|c|c|}
\hline \multirow[b]{3}{*}{$a_{0}$} & \multicolumn{9}{|c|}{ Variável Dependente: Horas Trabalhadas com Média Móvel (Set/1999-Out/2005) } \\
\hline & \multicolumn{3}{|c|}{ Modelo 1 } & \multicolumn{3}{|c|}{ Modelo 2} & \multicolumn{3}{|c|}{ Modelo 3} \\
\hline & 0,610 & $(0,537)$ & & 0,436 & 0,457 & & 1,026 & $(0,741)$ & \\
\hline$?_{1}$ & 0,470 & $(0,171)$ & $* * *$ & 0,639 & 0,159 & $* * *$ & 0,565 & $(0,185)$ & $* * *$ \\
\hline$?_{2}$ & 0,154 & $(0,189)$ & & 0,178 & 0,184 & & 0,249 & $(0,177)$ & \\
\hline$?_{3}$ & $-0,931$ & $(0,209)$ & $* * *$ & $-0,652$ & 0,180 & $* * *$ & $-0,736$ & $(0,191)$ & $* * *$ \\
\hline$?_{4}$ & 0,326 & $(0,199)$ & & 0,332 & 0,162 & $* *$ & 0,311 & $(0,197)$ & \\
\hline$?_{5}$ & $-0,062$ & $(0,217)$ & & & & & & & \\
\hline$?_{6}$ & $-0,482$ & $(0,203)$ & $* *$ & & & & & & \\
\hline$B_{0}$ & $-0,563$ & $(0,434)$ & & $-0,081$ & 0,230 & & $-0,448$ & $(0,194)$ & $* *$ \\
\hline$B_{1}$ & 0,329 & $(0,698)$ & & 0,100 & 0,276 & & $-0,940$ & $(0,496)$ & $*$ \\
\hline$\beta_{2}$ & $-0,401$ & $(0,726)$ & & 0,249 & 0,282 & & 1,518 & $(0,965)$ & \\
\hline$\beta_{3}$ & 0,597 & $(0,728)$ & & $-0,416$ & 0,325 & & $-0,628$ & $(1,060)$ & \\
\hline$\beta_{4}$ & $-0,645$ & $(0,809)$ & & $-0,123$ & 0,346 & & $-1,264$ & $(1,107)$ & \\
\hline$\beta_{5}$ & 0,412 & $(0,797)$ & & 0,363 & 0,325 & & 1,657 & $(1,304)$ & \\
\hline$\beta_{6}$ & 0,065 & $(0,731)$ & & $-0,153$ & 0,344 & & $-1,025$ & $(1,233)$ & \\
\hline$\beta_{7}$ & $-0,692$ & $(0,740)$ & & $-0,171$ & 0,330 & & $-0,453$ & $(1,234)$ & \\
\hline$\beta_{8}$ & 0,106 & $(0,763)$ & & $-0,144$ & 0,323 & & 1,743 & $(1,254)$ & \\
\hline$B_{9}$ & 0,573 & $(0,788)$ & & 0,616 & 0,311 & $* *$ & $-1,585$ & $(1,070)$ & \\
\hline$B_{10}$ & $-0,336$ & $(0,786)$ & & $-0,372$ & 0,260 & & 0,081 & $(1,024)$ & \\
\hline$\beta_{11}$ & 0,208 & $(0,733)$ & & $-0,022$ & 0,253 & & 0,604 & $(0,877)$ & \\
\hline$\beta_{12}$ & $-0,237$ & $(0,343)$ & & 0,420 & 0,220 & $*$ & $-0,166$ & $(0,408)$ & \\
\hline $\mathrm{d}_{0}$ & $-0,016$ & $(0,230)$ & & $-0,100$ & 0,117 & & 1,157 & $(0,531)$ & $* *$ \\
\hline $\mathrm{d}_{1}$ & 0,123 & $(0,534)$ & & 0,090 & 0,261 & & $-2,748$ & $(1,563)$ & $*$ \\
\hline $\mathrm{d}_{2}$ & 0,304 & $(0,613)$ & & 0,073 & 0,291 & & 2,187 & $(2,116)$ & \\
\hline$d_{3}$ & $-0,989$ & $(0,675)$ & & $-0,044$ & 0,281 & & 0,283 & $(2,014)$ & \\
\hline $\mathrm{d}_{4}$ & 0,634 & $(0,835)$ & & $-0,213$ & 0,306 & & $-2,211$ & $(2,338)$ & \\
\hline $\mathrm{d}_{5}$ & 0,243 & $(0,803)$ & & 0,279 & 0,319 & & 2,268 & $(2,506)$ & \\
\hline $\mathrm{d}_{6}$ & $-0,774$ & $(0,674)$ & & 0,036 & 0,304 & & $-0,532$ & $(2,350)$ & \\
\hline $\mathrm{d} 7$ & 0,422 & $(0,661)$ & & $-0,289$ & 0,302 & & $-2,122$ & $(2,464)$ & \\
\hline $\mathrm{d}_{8}$ & 0,457 & $(0,680)$ & & 0,085 & 0,300 & & 3,332 & $(2,262)$ & \\
\hline $\mathrm{d}_{9}$ & $-0,685$ & $(0,716)$ & & 0,128 & 0,254 & & $-1,775$ & $(1,975)$ & \\
\hline$d_{10}$ & 0,272 & $(0,782)$ & & $-0,052$ & 0,226 & & $-0,596$ & $(1,956)$ & \\
\hline$d_{11}$ & $-0,240$ & $(0,688)$ & & $-0,185$ & 0,208 & & 1,127 & $(1,389)$ & \\
\hline $\mathrm{d}_{12}$ & 0,247 & $(0,284)$ & & 0,176 & 0,100 & $*$ & $-0,416$ & $(0,464)$ & \\
\hline Observações & 60 & & & 60 & & & 56 & & \\
\hline$R^{2}$ & 0,806 & & & 0,766 & & & 0,809 & & \\
\hline Teste LM B-G & 0,307 & & & 0,617 & & & 0,617 & & \\
\hline
\end{tabular}

Sendo que *, ** e *** significa a variável é estatisticamente significante a $10 \%, 5 \%$ e $1 \%$, respectivamente.

Teste LM B-G (Breusch-Godfrey) de correlação serial, para a hipótese nula de não correlação serial (P-valor). 
Tabela 5. Resultado-Horas Trabalhadas (Degenerado)

\begin{tabular}{|c|c|c|c|c|c|c|c|c|c|}
\hline \multicolumn{10}{|c|}{ Variável Dependente: Horas Trabalhadas com Média Móvel (Set/1999-Out/2005) } \\
\hline & \multicolumn{3}{|c|}{ Modelo 1} & \multicolumn{3}{|c|}{ Modelo 2} & \multicolumn{3}{|c|}{ Modelo 3} \\
\hline$\alpha_{0}$ & 0,884 & $(0,353)$ & $* *$ & 0,786 & $(0,289)$ & $* * *$ & 0,953 & $(0,363)$ & $* * *$ \\
\hline$?_{1}$ & 0,546 & $(0,120)$ & $* * *$ & 0,586 & $(0,114)$ & $* * *$ & 0,405 & $(0,129)$ & $* * *$ \\
\hline$?_{2}$ & 0,110 & $(0,127)$ & & 0,237 & $(0,135)$ & $* *$ & 0,157 & $(0,131)$ & \\
\hline$?_{3}$ & $-0,472$ & $(0,127)$ & $* * *$ & $-0,658$ & $(0,129)$ & $* * *$ & $-0,357$ & $(0,118)$ & $* * *$ \\
\hline$?_{4}$ & 0,238 & $(0,118)$ & $* *$ & 0,358 & $(0,133)$ & $* * *$ & & & \\
\hline$? 5$ & $-0,057$ & $(0,065)$ & & & & & & & \\
\hline$?_{6}$ & $-0,037$ & $(0,017)$ & $* *$ & & & & & & \\
\hline$\beta_{1}$ & $-0,057$ & $(0,065)$ & & & & & $-0,166$ & $(0,083)$ & $* *$ \\
\hline$\beta_{4}$ & & & & 0,074 & $(0,140)$ & & & & \\
\hline$\delta_{1}$ & $-0,037$ & $(0,017)$ & $* *$ & & & & $-0,040$ & $(0,018)$ & $* *$ \\
\hline$\delta_{4}$ & & & & $-0,389$ & $(0,120)$ & $* * *$ & & & \\
\hline Observações & 72 & & & 68 & & & 67 & & \\
\hline $\mathrm{R}^{2}$ & 0,490 & & & 0,597 & & & 0,476 & & \\
\hline Teste LM B-G & 0,071 & & & 0,270 & & & 0,079 & & \\
\hline
\end{tabular}

Tabela 6. Resultado-Taxa de Desemprego

\begin{tabular}{|c|c|c|c|c|c|c|c|c|c|}
\hline \multicolumn{10}{|c|}{ Variável Dependente: Taxa de Desemprego com Média Móvel (Set/1999-Out/2005) } \\
\hline & \multicolumn{3}{|c|}{ Modelo 1} & \multicolumn{3}{|c|}{ Modelo 2} & \multicolumn{3}{|c|}{ Modelo 3} \\
\hline$\alpha_{0}$ & 0,566 & $(0,263)$ & $* *$ & 0,676 & $(0,312)$ & $* *$ & 0,549 & $(0,497)$ & \\
\hline$?_{1}$ & 2,062 & $(0,151)$ & $* * *$ & 2,375 & $(0,223)$ & $* * *$ & 2,363 & $(0,224)$ & $* * *$ \\
\hline$?_{2}$ & $-1,494$ & $(0,307)$ & $* * *$ & $-2,762$ & $(0,540)$ & $* * *$ & $-2,472$ & $(0,524)$ & $* * *$ \\
\hline$?_{3}$ & $-0,514$ & $(0,329)$ & & 1,863 & $(0,665)$ & $* * *$ & 0,849 & $(0,575)$ & \\
\hline$? 4$ & 1,814 & $(0,344)$ & $* * *$ & $-0,683$ & $(0,479)$ & & 1,011 & $(0,536)$ & $*$ \\
\hline$?_{5}$ & $-1,286$ & $(0,310)$ & $* * *$ & 0,102 & $(0,170)$ & & $-1,440$ & $(0,598)$ & $* *$ \\
\hline$?_{6}$ & 0,323 & $(0,137)$ & $* *$ & & & & 0,268 & $(0,550)$ & \\
\hline$?_{7}$ & & & & & & & 0,968 & $(0,527)$ & $*$ \\
\hline$?_{8}$ & & & & & & & $-0,886$ & $(0,527)$ & \\
\hline$?_{9}$ & & & & & & & $-0,125$ & $(0,386)$ & \\
\hline$?_{10}$ & & & & & & & 0,964 & $(0,378)$ & $* *$ \\
\hline$?_{11}$ & & & & & & & $-0,775$ & $(0,375)$ & $*$ \\
\hline$? 12$ & & & & & & & 0,193 & $(0,184)$ & \\
\hline
\end{tabular}




\section{Continuação da Tabela 6.}

\begin{tabular}{|c|c|c|c|c|c|c|c|c|c|}
\hline$\beta_{0}$ & 0,118 & $(0,119)$ & & 0,003 & $(0,088)$ & & $-0,043$ & $(0,055)$ & \\
\hline$\beta_{1}$ & $-0,323$ & $(0,221)$ & & 0,236 & $(0,091)$ & $* *$ & 0,034 & $(0,145)$ & \\
\hline$B_{2}$ & 0,182 & $(0,228)$ & & $-0,228$ & $(0,096)$ & $* *$ & 0,381 & $(0,212)$ & $*$ \\
\hline$B_{3}$ & 0,075 & $(0,219)$ & & 0,208 & $(0,109)$ & $*$ & $-0,469$ & $(0,227)$ & $*$ \\
\hline$B_{4}$ & 0,157 & $(0,233)$ & & $-0,062$ & $(0,111)$ & & 0,720 & $(0,280)$ & $* *$ \\
\hline$B_{5}$ & $-0,248$ & $(0,232)$ & & 0,108 & $(0,105)$ & & $-0,829$ & $(0,314)$ & $* *$ \\
\hline$B_{6}$ & $-0,018$ & $(0,213)$ & & $-0,111$ & $(0,111)$ & & 0,578 & $(0,309)$ & $*$ \\
\hline$B_{7}$ & 0,223 & $(0,216)$ & & 0,189 & $(0,109)$ & $*$ & $-0,178$ & $(0,324)$ & \\
\hline$B_{8}$ & $-0,155$ & $(0,223)$ & & $-0,125$ & $(0,113)$ & & 0,235 & $(0,341)$ & \\
\hline$B_{9}$ & 0,259 & $(0,226)$ & & 0,050 & $(0,106)$ & & $-0,216$ & $(0,272)$ & \\
\hline$B_{10}$ & $-0,064$ & $(0,217)$ & & 0,062 & $(0,083)$ & & 0,349 & $(0,241)$ & \\
\hline$\beta_{11}$ & $-0,256$ & $(0,199)$ & & 0,071 & $(0,080)$ & & $-0,315$ & $(0,221)$ & \\
\hline$\beta_{12}$ & 0,166 & $(0,091)$ & $*$ & 0,027 & $(0,079)$ & & 0,205 & $(0,130)$ & \\
\hline $\mathrm{d}_{0}$ & $-0,014$ & $(0,073)$ & & $-0,015$ & $(0,042)$ & & 0,049 & $(0,157)$ & \\
\hline $\mathrm{d}_{1}$ & 0,067 & $(0,165)$ & & $-0,053$ & $(0,092)$ & & $-0,525$ & $(0,379)$ & \\
\hline $\mathrm{d}_{2}$ & $-0,046$ & $(0,186)$ & & 0,244 & $(0,104)$ & $* *$ & 1,206 & $(0,453)$ & $*$ \\
\hline $\mathrm{d}_{3}$ & 0,141 & $(0,199)$ & & $-0,375$ & $(0,103)$ & $* * *$ & $-1,689$ & $(0,509)$ & $* * *$ \\
\hline $\mathrm{d}_{4}$ & $-0,341$ & $(0,234)$ & & 0,362 & $(0,113)$ & $* * *$ & 1,969 & $(0,654)$ & $* * *$ \\
\hline$d_{5}$ & 0,200 & $(0,227)$ & & $-0,255$ & $(0,115)$ & $* *$ & $-1,689$ & $(0,674)$ & $* *$ \\
\hline $\mathrm{d}_{6}$ & 0,098 & $(0,195)$ & & 0,123 & $(0,104)$ & & 0,951 & $(0,627)$ & \\
\hline $\mathrm{d}_{7}$ & $-0,184$ & $(0,197)$ & & $-0,059$ & $(0,099)$ & & $-0,473$ & $(0,703)$ & \\
\hline $\mathrm{d}_{8}$ & 0,266 & $(0,199)$ & & 0,103 & $(0,096)$ & & 0,467 & $(0,636)$ & \\
\hline $\mathrm{d}_{9}$ & $-0,280$ & $(0,208)$ & & $-0,111$ & $(0,085)$ & & $-0,649$ & $(0,483)$ & \\
\hline $\mathrm{d}_{10}$ & $-0,052$ & $(0,224)$ & & 0,107 & $(0,074)$ & & 0,757 & $(0,468)$ & \\
\hline $\mathrm{d}_{11}$ & 0,356 & $(0,196)$ & $*$ & $-0,093$ & $(0,065)$ & & $-0,608$ & $(0,362)$ & \\
\hline $\mathrm{d}_{12}$ & $-0,185$ & $(0,083)$ & $* *$ & 0,049 & $(0,034)$ & & 0,254 & $(0,134)$ & $*$ \\
\hline Observações & 60 & & & 60 & & & 56 & & \\
\hline $\mathrm{R}^{2}$ & 0,996 & & & 0,994 & & & 0,999 & & \\
\hline Teste LM B-G & 0,148 & & & 0,068 & & & 0,249 & & \\
\hline
\end{tabular}

Após essa exposição dos resultados, verificaremos se, em conjunto, os coeficientes das regressões são estatisticamente diferentes de zero (tabela 7), e posteriormente somaremos os coeficientes significativos na tabela 8. 
Tabela 7. Resultado - Taxa de Desemprego (Degenerado)

\begin{tabular}{|c|c|c|c|c|c|c|c|c|c|}
\hline \multirow{2}{*}{\multicolumn{3}{|c|}{$\begin{array}{r}\text { Variável Dependente: Taxa de } \\
\text { Modelo } 1\end{array}$}} & \multirow{2}{*}{\multicolumn{3}{|c|}{$\begin{array}{r}\text { Desemprego com Média } \\
\text { Modelo } 2 \\
\end{array}$}} & \multirow{2}{*}{\multicolumn{4}{|c|}{$\begin{array}{c}\text { Móvel (Set/1999-Out/2005) } \\
\text { Modelo } 3 \\
\end{array}$}} \\
\hline & & & & & & & & & \\
\hline$\alpha_{0}$ & 0,121 & $(0,256)$ & & 0,525 & $(0,284)$ & $*$ & 0,482 & $(0,232)$ & $* *$ \\
\hline$?_{1}$ & 2,207 & $(0,131)$ & $* * *$ & 1,964 & $(0,116)$ & $* * *$ & 2,176 & $(0,115)$ & $* * *$ \\
\hline$?_{2}$ & $-1,777$ & $(0,319)$ & $* * *$ & $-1,107$ & $(0,307)$ & $* * *$ & $-1,647$ & $(0,286)$ & $* * *$ \\
\hline$?_{3}$ & $-0,271$ & $(0,384)$ & & $-0,714$ & $(0,450)$ & & $-0,443$ & $(0,357)$ & \\
\hline$?_{4}$ & 1,832 & $(0,377)$ & $* * *$ & 1,162 & $(0,358)$ & $* * *$ & 1,977 & $(0,341)$ & $* * *$ \\
\hline$?_{5}$ & $-1,421$ & $(0,409)$ & $* * *$ & $-0,830$ & $(0,325)$ & $* *$ & $-1,376$ & $(0,378)$ & $* * *$ \\
\hline$? 6$ & 0,163 & $(0,366)$ & & 0,488 & $(0,338)$ & & $-0,428$ & $(0,411)$ & \\
\hline$?_{7}$ & 0,435 & $(0,356)$ & & $-0,030$ & $(0,137)$ & & 1,363 & $(0,377)$ & $* * *$ \\
\hline$? 8$ & $-0,358$ & $(0,294)$ & & & & & $-0,691$ & $(0,305)$ & $* *$ \\
\hline$?_{9}$ & 0,155 & $(0,119)$ & & & & & $-0,539$ & $(0,307)$ & $*$ \\
\hline$? 10$ & & & & & & & 1,080 & $(0,259)$ & $* * *$ \\
\hline$?_{11}$ & & & & & & & $-0,538$ & $(0,105)$ & $* * *$ \\
\hline$\beta_{3}$ & & & & & & & 0,043 & $(0,024)$ & $*$ \\
\hline$\beta_{4}$ & 0,040 & $(0,023)$ & $*$ & & & & & & \\
\hline$\beta_{10}$ & & & & 0,151 & $(0,041)$ & $* * *$ & & & \\
\hline$\delta_{3}$ & & & & & & & 0,016 & $(0,006)$ & $* * *$ \\
\hline$\delta_{4}$ & 0,014 & $(0,007)$ & $* *$ & & & & & & \\
\hline$\delta_{10}$ & & & & 0,012 & $(0,007)$ & $*$ & & & \\
\hline Observações & 68 & & & 62 & & & 65 & & \\
\hline $\mathrm{R}^{2}$ & 0,987 & & & 0,986 & & & 0,992 & & \\
\hline Teste LM B-G & 0,188 & & & 0,235 & & & 0,995 & & \\
\hline
\end{tabular}

Na tabela 7, apresentamos os resultados de testes de Wald para a hipótese nula de que os coeficientes são iguais a zero. A hipótese nula é rejeitada para os três modelos apenas quando a variável explicada é o desemprego. Para a produção industrial, somente nos modelos 1 e 3 rejeitamos a hipótese dos coefic ientes serem iguais a zero para a parte esperada da taxa de juros, e para horas trabalhadas não rejeitamos em nenhum modelo a hipótese dos coeficientes serem iguais a zero. 
Tabela 8. Teste Wald

\begin{tabular}{lccc}
\hline \hline & Teste Wald* para os Coeficientes das Tabelas 1, 3 e 5 & \\
\hline & Produção Industrial & Horas Trabalhadas & Desemprego \\
\hline Modelo 1 & & & \\
Não Esperada & 14,777 & & \\
P-Valor & 0,321 & 11,426 & 28,529 \\
Esperada & 25,357 & 0,575 & 0,008 \\
P-Valor & 0,021 & 14,000 & 26,841 \\
& & 0,374 & 0,013 \\
Modelo 2 & & & \\
Não Esperada & 11,564 & 14,691 & 29,450 \\
P-Valor & 0,564 & 0,327 & 0,006 \\
Esperada & 15,743 & 14,341 & 31,226 \\
P-Valor & 0,263 & 0,350 & 0,003 \\
& & & \\
Modelo 3 & 17,506 & 16,207 & 6,031 \\
Não Esperada & 0,177 & 0,238 & 0,000 \\
P-Valor & 24,938 & 10,058 & 57,573 \\
Esperada & 0,024 & 0,689 & 0,000 \\
P-Valor & & & \\
\hline \hline
\end{tabular}

* Para a Hipót ese Nula de que os coeficientes são iguais a zero.

Para ilustrar os efeitos de uma surpresa monetária por nós encontrados, suponhamos, usando um exemplo concreto, que a maioria dos agentes do mercado esteja prevendo um corte na taxa Selic de 0,50\%, com um grupo menor fechando aposta em um corte de apenas 0,25\%. Admitamos que a média ponderada destes valores seja, digamos, de $0,40 \%^{12}$. Desta forma, caso o corte seja efetivamente de $0,50 \%$, haveria um erro de previsão do mercado de $-0,10 \%$.

Segundo nossas es timativas, esse erro de previsão - juros caindo mais do que o esperado causaria uma redução de $0,038 \%$ na taxa de desemprego ao longo de um ano, considerando apenas o efeito da parte não-esperada dos juros. O efeito da parte esperada seria de $-0,021 \%$, ou seja, quase a metade do efeito atribuído para o caso da surpresa de juros ${ }^{13}$.

\footnotetext{
${ }^{12}$ De acordo com um estudo feito pelo próprio Banco Central do Brasil, o erro de previsão para a nova taxa Selic é em torno de 0,10\%, o mesmo utilizado no exemplo. Relatório de Inflação (2006).
} 
Tabela 9. Soma dos Coeficientes

\begin{tabular}{|c|c|c|c|c|}
\hline \multicolumn{5}{|c|}{ Soma dos Coeficientes Significantes das Tabelas 1,3 e 5} \\
\hline & $\mathbf{P}$-valor $<$ & Produção Industrial & Horas Trabalhadas & Desemprego \\
\hline \multicolumn{5}{|l|}{ Modelo 1} \\
\hline \multirow{3}{*}{ Não Esperada } & $10 \%$ & -2.604 & - & 0,166 \\
\hline & $5 \%$ & -2.604 & - & - \\
\hline & $1 \%$ & 3.899 & - & - \\
\hline \multirow[t]{3}{*}{ Esperada } & $10 \%$ & -3.469 & - & 0,171 \\
\hline & $5 \%$ & -3.469 & - & $-0,185$ \\
\hline & 1 & -5.774 & - & \\
\hline \multicolumn{5}{|l|}{ Modelo 2} \\
\hline \multirow[t]{3}{*}{ Não Esperada } & 10 & -1.107 & 1.036 & 0,405 \\
\hline & 5 & - & 0,616 & 0,008 \\
\hline & 1 & - & - & - \\
\hline \multirow[t]{3}{*}{ Esperada } & 10 & - & 0,176 & $-0,025$ \\
\hline & 5 & - & - & $-0,025$ \\
\hline & 1 & - & - & 0,013 \\
\hline \multicolumn{5}{|l|}{ Modelo 3} \\
\hline \multirow[t]{3}{*}{ Não Esperada } & 10 & -2.801 & -1.389 & 0,382 \\
\hline & 5 & - & $-0,448$ & $-0,109$ \\
\hline & 1 & - & - & - \\
\hline \multirow[t]{3}{*}{ Esperada } & 10 & -6.177 & -1.592 & 0,052 \\
\hline & 5 & - & 1.157 & -1.409 \\
\hline & 1 & - & - & $-0,280$ \\
\hline
\end{tabular}

No caso da produção industrial para regressões degeneradas, os três modelos se mostraram semelhantes, e, analisando apenas o terceiro, teríamos um impacto na produção já no primeiro mês após uma queda de juros maior que a esperada. Esse movimento inesperado causaria uma variação na taxa de crescimento da produção de $0,043 \%$. O impacto da mudança esperada seria bem menor, de $0,024 \%$.

Vale notar que neste trabalho encontramos uma resposta real à surpresa de juros (e também da parte esperada) muito mais rápida do que o usual. Em alguns trabalhos empíricos, como Romer e Romer (2003), e Bernanke e Mihov (1998), usando da dos da economia americana, a

\footnotetext{
${ }^{13}$ Resultados obtidos para o modelo 3, uma vez que esse obteve o melhor desempenho para todos os casos.
} 
resposta é sempre mais lenta, dando-se em geral apenas a partir do sexto mês da alteração na política monetária ${ }^{14}$.

Os resultados obtidos aqui também podem ser cotejados aos de Barro (1978) e Mishkin (1982). Como nestes, também encontramos a evidência de que o efeito de mudanças da política monetária sobre a taxa do desemprego vem em defasagens mais longas que no caso da produção. No entanto, diferentemente de Barro, encontramos uma resposta tanto da parte não-antecipada da mudança de juros, como da parte antecipada, principalmente para a variável desemprego, que foi a variável inicial de estudo daquele autor na década de 70 .

\footnotetext{
${ }^{14}$ Ambos usam a metodologia VAR (Vetor Auto-Regressivo).
} 


\section{COMENTÁRIOS FINAIS}

Neste trabalho contribuímos para a discussão sobre os impactos da política monetária nas variáveis reais da economia brasileira. Nossa contribuição consiste em testar o efeito das surpresas de política monetária sobre a atividade econômica e o desemprego, com base nas predições teóricas dos modelos neoclássicos e novo-keynesianos de que o que importa para o comportamento das variáveis reais é a parte nãoesperada da política monetária.

Os resultados encontrados indicam que a parte não-esperada da taxa de juros afeta sim o comportamento de variáveis, como desemprego e atividade industrial, em concordância com o explicitado em nível teórico. Além disso, nossas estimações mostram que o efeito da parte não-esperada da mudança de política monetária é quantitativamente mais relevante do que o da parte esperada.

O segundo ponto a destacar diz respeito à velocidade da resposta a um choque monetário, que se mostrou muito maior do que a previamente imaginada. Finalmente, como encontrado em outros estudos, a resposta da produção precede a da taxa de desemprego. 


\section{REFERÊNCIAS:}

Barro, R. (1977). Unanticipated money growth and unemployment in the United States. The American Economic Review, v. 67, n. 2, p. 101-115, Mar. 1977.

, (1978). Unanticipated money, output, and the price level in the United States.

Journal of Political Economy, v. 86, n. 4, p. 549-580, Ago. 1978.

Bernanke, B. \& Mihov, I. (1998). Measuring Monetary Policy. The Quarterly Journal of Economics, v. 113, n. 3, p. 869-902, Ago. 1998.

Davidson, R. \& MacKinnon, J. (2004). Econometric Theory and Methods. Oxford, Inglaterra: Oxford University Press, 2004.

Favero, C. (2001). Applied Macroeconometrics. Oxford, Inglaterra: Oxford University Press, 2001.

Greene, W. (2003). Econometrics Analysis. New Jersey, EUA: Prentice Hall, $5^{\mathrm{a}}$ ed. Primeira Ediçao 1993.

Lucas, R. (1973). Some international evidence on output-inflation trade-offs. The American Economic Review, v. 63, n. 3, p. 326-334, Jun. 1973.

Mishkin, F. (1982). Does anticipated aggregate demand policy matter? Further econometric results. American Economic Review, v. 72, n. 4, p. 788-802, Set. 1982. 
Relatório de Inflação (2006). Banco Central do Brasil, volume 8, número 1, p. 1-165, Mar. 2006. (http://www.bcb.gov.br/?RELINF200603)

Romer, D. (2001). Advanced Macroeconomics. Nova York, EUA: McGraw-Hill, $2^{\mathrm{a}}$ ed.

Romer, D. \& Romer, C. (2003). A New Measure of Monetary Shocks: Derivation and Implications. NBER, Working Paper 9866.

Taylor, J. (1993) Discretion versus Policy Rules in Practice, Carnegie-Rochester Conference Series on Public Policy, 39, p. 195-214, 1993.

, (1999). The robustness and efficiency of monetary policy rules as guidelines for interest rate setting by the European Central Bank. Journal of Monetary Economics, v. 43, p. 655-679, 1999. 